\title{
Proteomic profiling of chronic low-frequency stimulated fast muscle
}

\author{
Pamela Donoghue ${ }^{1}$, Phil Doran ${ }^{2}$, Kieran Wynne ${ }^{1}$, Kasper Pedersen ${ }^{1}$, \\ Michael J. Dunn ${ }^{1}$ and Kay Ohlendieck ${ }^{2}$ \\ ${ }^{1}$ Proteome Research Centre, UCD Conway Institute, University College Dublin, Dublin, Ireland \\ ${ }^{2}$ Department of Biology, National University of Ireland, Maynooth, Co. Kildare, Ireland
}

\begin{abstract}
Skeletal muscle fibre transitions occur in many biological processes, in response to alterations in neuromuscular activity, in muscular disorders, during age-induced muscle wasting and in myogenesis. It was therefore of interest to perform a comprehensive proteomic profiling of muscle transformation. Chronic low-frequency stimulation of the rabbit tibialis anterior muscle represents an established model system for studying the response of fast fibres to enhanced neuromuscular activity under conditions of maximum activation. We have conducted a DIGE analysis of unstimulated control specimens versus 14- and 60-day conditioned muscles. A differential expression pattern was observed for 41 protein species with 29 increased and 12 decreased muscle proteins. Identified classes of proteins that are changed during the fast-to-slow transition process belong to the contractile machinery, ion homeostasis, excitation-contraction coupling, capillarization, metabolism and stress response. Results from immunoblotting agreed with the conversion of the metabolic, regulatory and contractile molecular apparatus to support muscle fibres with slower twitch characteristics. Besides confirming established muscle elements as reliable transition markers, this proteomics-based study has established the actin-binding protein cofilin-2 and the endothelial marker transgelin as novel biomarkers for evaluating muscle transformation.
\end{abstract}

\section{Keywords:}

Chronic low-frequency stimulation / DIGE / Fibre transition / Muscle plasticity / Skeletal muscle proteomics

\section{Introduction}

One of the major aims of modern physiology and biochemistry is to understand the integrated function of complex biological systems. The study of skeletal muscle fibers represents an especially challenging area of biological research due to the enormous cellular plasticity of this type of

Correspondence: Professor Dr. Kay Ohlendieck, Department of Biology, National University of Ireland, Maynooth, Co. Kildare, Ireland

E-mail: kay.ohlendieck@nuim.ie

Fax: +353-1-708-3845

Abbreviation: SSA, same spot analysis
Received: March 20, 2007

Revised: May 28, 2007

Accepted: June 11, 2007 tissue [1-4]. Conventional molecular biological and biochemical methods, traditionally used to investigate physiological mechanisms, are often limited to studying only a few selected genes or proteins. In contrast, the emergence of high-throughput genomics and proteomics methods has opened up new possibilities for more comprehensive approaches of determining biological processes [5, 6]. MSbased proteomics technologies promise to be extremely useful in the global cataloguing and quantifying of individual protein species. The proteomics of cardiac muscle is an excellent example of a new protein biochemical field that has made enormous progress [7], since detailed heart protein reference maps are now available [8]. Due to its more heterogeneous nature, the proteomic profiling of skeletal muscle fibres is still in its infancy, but has already made sub- 
stantial contributions to our general understanding of basic and applied myology. Recent studies have successfully cataloged the skeletal muscle proteome from various animal species [9-14], determined global differences in protein expression between predominantly slow- versus fast-twitching fibres [15-18], and have identified novel marker proteins of muscle growth [19], myoblast differentiation [20], neonatal fibre necrosis [18], hypertrophy [21], muscular dystrophy [2226], dysferlinopathy [27], immobilization-induced atrophy [28, 29] and ageing-induced sarcopenia [30-34]. In analogy, based on the findings of an initial proteomic analysis of the fast-to-slow fibre transformation process using a conventional nonfluorescent method [35], this report describes the detailed DIGE analysis of the differential expression of the fast skeletal muscle proteome following chronic low-frequency stimulation.

Skeletal muscle plasticity plays a central role in the adaptive response to changed physiological demands [2]. Predominantly fast-twitching muscle fibers convert to slower fibers in response to enhanced neuromuscular activity [4], whereby muscle conditioning has been instrumental for the development of alternative biomedical treatments such as dynamic cardiomyopathy [36], as well as the enhancement of our biological understanding of tissue transdifferentiation [37]. Chronic electrostimulation of fast muscles results in an improved resistance to fatigue, which is reflected physiologically by an increase in the time-topeak twitch tension and half-relaxation time [38]. An elevation of aerobic-oxidative capacity, decreased fibre calibre and changes in the density of distinct muscle proteins, switches in isoform expression patterns and alterations in proteinprotein interactions are established biochemical and cell biological hallmarks of transformed skeletal muscles. This includes: (i) metabolic pathways such as the citric acid cycle, fatty acid oxidation and the respiratory chain which causes a drastic increase in enzymes of aerobic substrate oxidation $[39,40]$, (ii) the contractile apparatus that undergoes a stepwise replacement of myosin light and heavy chains from fast isoforms to their slower counterparts [41, 42], (iii) the ion-regulatory machinery of the excitation-contractionrelaxation cycle with a shift from fast to slower isoforms with respect to the SERCA-type $\mathrm{Ca}^{2+}$-ATPases, the voltagesensing dihydropyridine receptor, the ryanodine receptor $\mathrm{Ca}^{2+}$-release channel and various $\mathrm{Ca}^{2+}$-binding proteins [43, 44], (iv) the neuromuscular junction with distinct changes in the acetylcholinesterase and the nicotinic acetylcholine receptor $[45,46]$ and, (v) a decrease in the supramolecular interaction pattern between $\mathrm{Ca}^{2+}$-regulatory proteins of the sarcoplasmic reticulum [47].

Here, we have investigated the muscle adaptation process in response to enhanced neuromuscular activity by MSbased proteomics, which has resulted in the identification of a number of new biological markers of the fast-to-slow transition process, such as cofilin-2 and transgelin. In the longterm, the detailed cell biological and physiological characterization of the role of these muscle proteins in fibre transdif- ferentiation may improve diagnostic procedures and/or the design of future strategies to treat muscle pathologies that involve fibre transformation.

\section{Material and methods}

\subsection{Materials}

CyDye DIGE fluor minimal dyes Cy2, Cy3 and Cy5, electrophoresis grade chemicals, IPG strips of pH 3-10 and IPG buffer of $\mathrm{pH}$ 3-10 for IEF and ACN were purchased from GE Healthcare (Little Chalfont, Bucks, UK). For peptide generation, sequencing grade-modified trypsin was obtained from Promega (Madison, WI). Ultrapure Protogel acrylamide stock solutions were from National Diagnostics (Atlanta, GA). Primary antibodies were from Affinity Bioreagents, Golden, CO (mAb IIH11 to the fast SERCA1 isoform of the $\mathrm{Ca}^{2+}$-ATPase; mAb IID8 to the slow SERCA 2 isoform of the $\mathrm{Ca}^{2+}$-ATPase), Sigma, Dorset, UK (mAb MY-32 to the fast $\mathrm{MHC}_{\mathrm{f}}$ isoforms IIA, IIB, IID of the myosin heavy chain (MHC); mAb NOQ7.54D to the slow $\mathrm{MHC}_{\mathrm{s}}$ isoform of the MHC), Abcam, Cambridge, UK (pAb ab14133 to cofilin-2; pAb ab11061 to $\alpha$ B-crystallin; $m A b$ ab21128-9F3 to cardiac fatty acid binding protein FABP-3) and Everest Biotech, Upper Heyford, Oxfordshire, UK (pAb EB06281 to transgelin/SM22-alpha). Peroxidase-conjugated secondary antibodies were purchased from Chemicon International (Temecula, CA, USA). Protease inhibitors, Immobilon NC membranes and chemiluminescence substrates were purchased from Roche Diagnostics (Mannheim, Germany) and Pierce and Warriner (Chester, UK), Millipore (Bedford, MA), respectively. All other chemicals used were of analytical grade and obtained from Sigma Chemical Company, Dorset, UK.

\subsection{Animal model}

The rabbit tibialis anterior muscle represents a predominantly fast-twitching muscle that is composed of four defined fibre types (I, IIC, IIA and IIB) [48]. Conditioning of the left hind limb of adult male New Zealand white rabbits was achieved through chronic tele-stimulation of the peroneal nerve [38]. Low frequency stimulation was performed at $10 \mathrm{~Hz}$ for 14 or 60 days $(n=3)$ in the Animal Facility of the University of Konstanz (Germany) under the direction of Dr. Dirk Pette (Professor Emeritus of Biology). The Irish Higher Education Authority and the Deutscher Akademischer Austauschdienst generously supported laboratory visits to Germany by the Maynooth team. Whole muscle specimens were dissected from the stimulated left hind limb and the unstimulated right hind limb and then quick-frozen in liquid nitrogen. Muscle samples were stored at $-80^{\circ} \mathrm{C}$ and shipped on dry ice prior to proteomics analysis. 


\subsection{Sample preparation}

Skeletal muscle tissue ( $250 \mathrm{mg}$ ) was ground into a fine powder using liquid nitrogen and immediately added to $1 \mathrm{~mL}$ of DIGE-compatible lysis buffer (9.5 M Urea, 2\% w/v CHAPS, $20 \mathrm{mM}$ Tris, $\mathrm{pH}$ 8.0-8.5) and incubated at room temperature for $1 \mathrm{~h}$. In order to maximize protein yields, protein samples were sonicated on ice for $3 \times 10 \mathrm{~s}$ bursts [26]. Following centrifugation at $14000 \times g_{\mathrm{av}}$ for $30 \mathrm{~min}$, protein fractions were collected, aliquoted and stored at $-80^{\circ} \mathrm{C}$ until further use. All preparative steps were performed at $0-4^{\circ} \mathrm{C}$ in the presence of a protease inhibitor cocktail $[26,35]$ to prevent excess proteolytic degradation. Protein concentration was determined using the Bradford dye binding assay [49].

\subsection{DIGE labelling}

Minimal CyDye labelling (GE Healthcare) was performed at a ratio of $25 \mu \mathrm{g}$ of protein/200 pmol of CyDye for 2-DE. Labelled samples were incubated on ice for $30 \mathrm{~min}$ in the dark. The labelling reaction was terminated by the addition of $10 \mathrm{mM}$ lysine. After a brief centrifugal spin, samples were incubated on ice in the dark for $10 \mathrm{~min}$ [26]. Labelled protein fractions were utilized immediately for further analysis or otherwise stored at $-20^{\circ} \mathrm{C}$. Protein samples were combined with an equal volume of $2 \times$ lysis buffer $(9.5 \mathrm{M}$ urea, $2 \% \mathrm{w} / \mathrm{v}$ CHAPS, $2 \% \mathrm{w} / \mathrm{v}$ DTT and $1.6 \% \mathrm{w} / \mathrm{v}$ Pharmalyte $\mathrm{pH} 3-10$ ) followed by in-gel rehydration overnight prior to IEF [26].

\subsection{2-DE}

Skeletal muscle extracts were diluted in rehydration solution (8 M urea, 0.5\% w/v CHAPS, 0.2\% w/v DTT and 0.2\% w/v Pharmalyte $\mathrm{pH} 3-10$ ) and applied to $24 \mathrm{~cm}$ IPG strips (pH 3-10, nonlinear (NL), GE Healthcare). Strips were rehydrated overnight at room temperature using an in-gel rehydration method [50]. The sample was then subjected to IEF at $0.05 \mathrm{~mA} / \mathrm{IPG}$ strip for $72000 \mathrm{Vh}$ at $20^{\circ} \mathrm{C}$. The strips were equilibrated in $6 \mathrm{M}$ urea, containing $30 \% \mathrm{v} / \mathrm{v}$ glycerol, $2 \% \mathrm{w} / \mathrm{v}$ SDS, $0.05 \mathrm{M}$ Tris-HCl, pH 8.8 and $0.01 \% \mathrm{w} / \mathrm{v}$ bromophenol blue with the addition of $1 \% \mathrm{w} / \mathrm{v}$ DTT for $15 \mathrm{~min}$. Subsequently, the strips were equilibrated in the same buffer without DTT but with the addition of $4.8 \% \mathrm{w} / \mathrm{v}$ iodoacetamide for $15 \mathrm{~min}$ [51]. The second dimension was carried out overnight using a Protean Plus Dodeca Cell system (BioRad Laboratories, Hemel Hempstead, Herts, UK) at $1 \mathrm{~W} / g e l$ at $15^{\circ} \mathrm{C}$ and was terminated when the dye front had just migrated off the lower end of the gels. A total of 12 gels were analysed with six DIGE gels representing the $0-14 \mathrm{~d}$ and six gels the 0-60d changes. Gels were subsequently scanned using a Typhoon variable mode imager 9400 (GE Healthcare), with a standard pixel volume of 40000-60000 for all scans.

\subsection{Expression analysis}

Following chronic low-frequency stimulation, expression changes in muscle proteins were determined by the protein expression analysis software programme Progenesis (Nonlinear Dynamics, Newcastle upon Tyne, UK). Prior to analysis, individual gels were warped to a single master gel. Since warping aligns protein spots on all analysed gels, this procedure facilitated spot-matching in downstream analysis. Gels imported into Progenesis were matched and normalized with nonspots filtered out on the basis of normalized spot volume. Prior to ANOVA testing $(p<0.01)$, same spot analysis (SSA) was used across all gels. SSA confers the same spot outline to corresponding spots in all gels in the series, eliminating experimental variation between spots. Proteins found to be significant were picked for tryptic digestion from Plus-One silver-stained micropreparative gels.

\subsection{MS analysis}

Tryptic digests were separated and analysed using a C-18 RPcolumn (Unitech, Dublin, Ireland) nano-LC system from Proxeon Biosystems (Odense, Denmark) connected directly to an LTQ Linear IT Mass spectrometer (Thermo Finnegan, Hemel Hempstead, Herts, UK). The samples were eluted from the C18 precolumn (Proxeon) by an increasing MeCN gradient $(0-90 \%)$. Generated mass spectra were searched against the UNIPROT database (release 7.6) and data collected by the Proline Proteomics Pipeline (Gerard Cagney Laboratory, UCD Conway Institute) utilizing the X! Tandem 2005.02.01 protein search engine. Parent mass error was -0.5 to $4.0 \mathrm{~m} / \mathrm{z}$ with fragment error of $0.4 \mathrm{Da}$. One missed tryptic cleavage site was accepted per peptide.

\subsection{Validation}

Validation of selected proteins identified by MS/MS analysis was carried out by 1-D immunoblot analysis, as previously described in detail [52]. The electrophoretic transfer of proteins to NC membranes was performed by the method of Towbin et al. [53]. Primary antibodies to the fast and slow MHC, the fast and slow sarcoplasmic reticulum $\mathrm{Ca}^{2+}$. ATPase, cofilin-2, $\alpha$ B-crystallin, the cardiac fatty acid binding-protein FABP-3 and transgelin were used to determine stimulation-induced changes in the abundance and/or isoform expression patterns of key muscle proteins.

\section{Results}

\subsection{Severely altered protein expression pattern in chronic low-frequency stimulated tibialis anterior muscle}

The comparative gel electrophoretic analysis of unstimulated versus 14- and 60-day electrostimulated fast muscles revealed 
a drastic change in the 2-D protein separation pattern of crude tissue extracts following fibre conditioning. The 2-D spot pattern of silver-stained gels was shown to be very comparable to published studies on the position of 2-D landmark species, such as contractile proteins [9-18], but the stimulated specimens differed considerably to the untreated control (Figs. 1A, C and E). Building on the recent successful identification of novel biomarkers for X-linked muscular dystrophy by DIGE analysis [26], we have here applied the same fluorescent technology to the comprehensive screen-
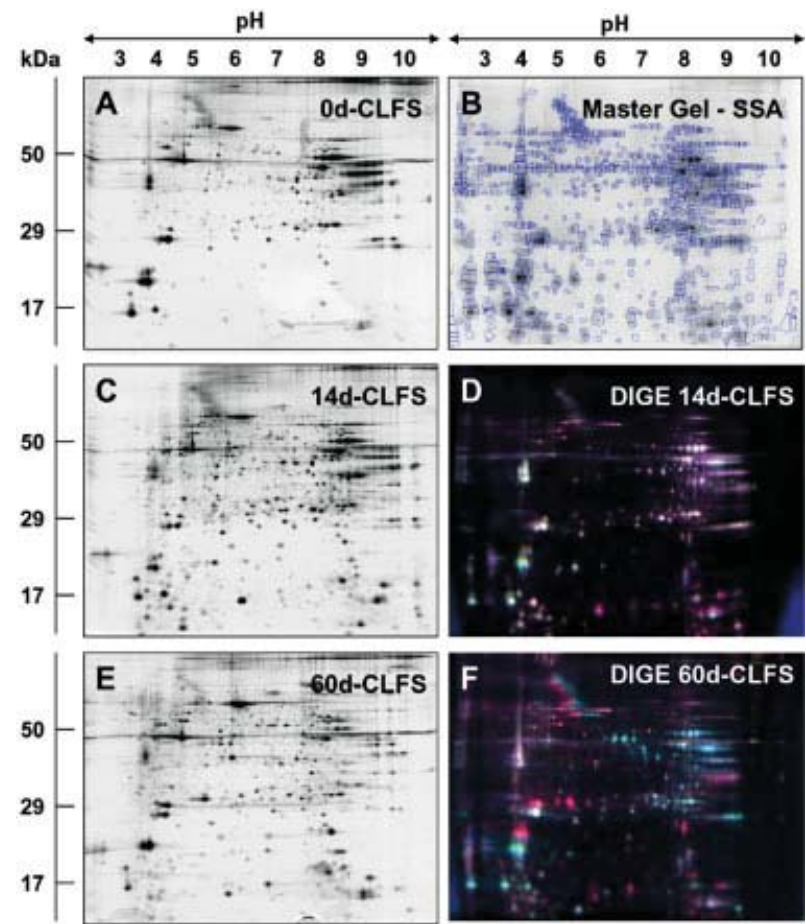

Figure 1. 2-D gel electrophoretic analysis of chronic low-frequency stimulated fast muscle. Shown are silver-stained 2-D gels of unstimulated ( $A ; 0 d)$, 14-day chronic low-frequency stimulated (C; 14d CLFS) and 60-day stimulated (E; 60d CLFS) fast muscles. The results of the SSA approach used in the Progenesis 2-D software analysis programme is shown in panel $B$. The image is the overlay of DIGE-labelled muscle extracts following 0,14 and 60 day stimulation. Panels $D$ and $F$ illustrate the overlay image of the CyDye fluors Cy2, Cy3 and Cy5, whereby in both sets Cy2 represents the pooled standard, Сy3 the unstimulated control sample and Cy5 the 14- or 60-day electrostimulated specimens, respectively. Shown are DIGE-labelled 2-D gels of unstimulated versus 14-day chronic low-frequency stimulated muscle (D; 14dCLFS), and unstimulated versus 60-day conditioned fast muscle (F; 60d-CLFS). IEF has been carried out on $24 \mathrm{~cm} \mathrm{pH} \mathrm{3-10} \mathrm{IPG}$ strips in the first dimension and standard $12 \%$ polyarcylamide slab gels in the second dimension. The $\mathrm{pH}$-values of the first dimension gel system and molecular mass standards (in kDa) of the second dimension are indicated on the top and on the left of the panels, respectively. See Figs. 2 and 4 for enlarged images of individual marker proteins with a changed abundance following chronic low-frequency stimulation, and Table 1 for a detailed listing of skeletal muscle proteins with a stimulation-induced change in their abundance as judged by DIGE analysis. ing of chronic low-frequency stimulated fast muscle. Although 2-D gels do not represent the entire muscle protein complement, gel electrophoretic techniques are indispensable tools for the biochemical profiling of complex proteomes [54], especially in conjunction with modern MS methods [55]. The differential fluorescent tagging of individual sets of proteins and separation within the same gel electrophoretic system [56-58] has greatly reduced gel-to-gel variation, and this advantage over other comparative proteomic approaches was exploited in this investigation of the muscle transformation process. As illustrated in Figs. 1D and F, the overlay images of the CyDye fluors Cy2, Cy3 and Cy5, representing the pooled standard, the unstimulated control sample and the 14- or 60-day electrostimulated specimens, respectively, clearly demonstrated large changes in the expression pattern of the conditioned fast skeletal muscle proteome. Since the SSA approach used in the Progenesis 2-D software analysis package imparts the same spot outline between corresponding spots in all investigated gels, the SSA system was employed. Figure 1B shows the image of the overlay of DIGE-labelled muscle extracts following 0,14 and 60 day stimulation, including the pattern of marked protein spots. Out of an original population of over 2700 2-D spots, 985 protein spots were chosen for SSA handling after filtering and spot editing. Proteins of interest, which had been highlighted by expression analysis, were picked from preparative gels for downstream analysis by ESI-MS/ MS.

\subsection{Confirmation of fast-to-slow muscle transition by contractile marker analysis}

In parallel to the in-depth analysis of the differential protein expression profile of stimulated fast muscles using the DIGE technique, a successful skeletal muscle transformation was confirmed by the evaluation of key contractile protein isoforms. It is well established that the various light and heavy chains of myosin undergo a stepwise replacement from fast to slow isoforms during fibre type shifting [41, 42], which has also been shown to occur in the case of regulatory elements of the contractile apparatus, such as the individual subunits of troponin [4]. The expanded views of 2-D landmark spots shown in Fig. 2 clearly demonstrated that the continuous telestimulation of the peroneal nerve at a frequency of $10 \mathrm{~Hz}$ has induced a fast-to-slow transition process. While a fast-twitch marker protein, such as the fast isoform of Troponin-I, exhibited a drastic reduction following electrostimulation (Fig. 2A), the expression of the slow isoform of Troponin-T was dramatically increased (Fig. 2B). The gradual replacement of fast with slow isoforms was also clearly documented by distinct alterations in the 2-D spot cluster that represents myosin light chains. The four isoforms of interest are individually labelled in Fig. 2C and clearly illustrated the gradual fast-to-slow transition process on the molecular level. 
Table 1. List of protein species with a changed expression level following chronic low-frequency stimulation of the rabbit tibialis anterior muscle

\begin{tabular}{|c|c|c|c|c|c|c|c|c|c|}
\hline $\begin{array}{l}\text { Spot } \\
\text { no. }\end{array}$ & $\begin{array}{l}\text { Accession } \\
\text { no. }\end{array}$ & Protein & $\begin{array}{l}\text { Peptides } \\
\text { matched }\end{array}$ & Peptide sequence & Charge & Score & $\begin{array}{l}0-14 d \\
\text { CLFS }\end{array}$ & $\begin{array}{l}0-60 d \\
\text { CLFS }\end{array}$ & $\begin{array}{l}14 d-60 d \\
\text { CLFS }\end{array}$ \\
\hline 1 & P01948 & Hemoglobin & 11 & $\begin{array}{l}\text { VNVEEVGGEALGR } \\
\text { VVAGVANALAHK } \\
\text { VNVEEVGGEAL } \\
\text { VVAGVANALAH } \\
\text { LLVVYPWTQR } \\
\text { SAVTALWGK } \\
\text { LSELHCDK } \\
\text { EFTPQVOAAYQK } \\
\text { LHVDPENFR } \\
\text { VHLSSEEK } \\
\text { VVAGVANAL }\end{array}$ & $\begin{array}{l}2 \\
2 \\
2 \\
2 \\
2 \\
2 \\
2 \\
2 \\
2 \\
2 \\
1\end{array}$ & -100.3 & 5.197 & 9.138 & 1.758 \\
\hline 2 & P05413 & $\begin{array}{l}\text { Cardiac fatty acid } \\
\text { binding protein FABP-3 }\end{array}$ & 9 & $\begin{array}{l}\text { WDGQETTLVR } \\
\text { SLGVGFATR } \\
\text { SLGVGFA } \\
\text { GOETTLVR } \\
\text { SIVTLDGGK } \\
\text { NGDILTLK } \\
\text { SLGVGF } \\
\text { NFDDYMK } \\
\text { NTEISFK }\end{array}$ & $\begin{array}{l}2 \\
2 \\
1 \\
2 \\
1 \\
1 \\
1 \\
1 \\
1\end{array}$ & -65.1 & 6.220 & 8.779 & 1.411 \\
\hline 3 & P04792 & HSP-27 & 4 & $\begin{array}{l}\text { QLSSGVSEIR } \\
\text { QDEHGYISR } \\
\text { DGVVEITGK } \\
\text { TKDGVVEITGK }\end{array}$ & $\begin{array}{l}2 \\
2 \\
1 \\
2\end{array}$ & -23.9 & 2.499 & 8.322 & 3.330 \\
\hline 4 & $09 Y 281$ & Cofilin-2 & 5 & $\begin{array}{l}\text { YALYDATYETK } \\
\text { ASGVTVNDEVIK } \\
\text { MIYASSK } \\
\text { WAPESAPLK } \\
\text { LLPLNDCR }\end{array}$ & $\begin{array}{l}2 \\
2 \\
1 \\
2 \\
2\end{array}$ & -39.4 & 2.455 & 7.594 & 3.094 \\
\hline 5 & P08590 & $\begin{array}{l}\text { Myosin light chain } \\
\text { MLC1 slow }\end{array}$ & 9 & $\begin{array}{l}\text { ALGQNPTQAEVLR } \\
\text { ITYGQCGDVLR } \\
\text { GQNPTOAEVLR } \\
\text { HVLATLGER } \\
\text { PTQAEVLR } \\
\text { EAFMLFDR } \\
\text { LMAGOEDSNG } \\
\text { APAPP } \\
\text { EGNGTVMGAELR }\end{array}$ & $\begin{array}{l}2 \\
2 \\
2 \\
2 \\
2 \\
2 \\
2 \\
1 \\
2\end{array}$ & -72.2 & 1.748 & 6.626 & 3.790 \\
\hline 6 & P12075 & Cytochrome- $c$ oxidase & 2 & $\begin{array}{l}\text { SGGGVPTDEEQATGLER } \\
\text { CPNCGTHYK }\end{array}$ & $\begin{array}{l}2 \\
2\end{array}$ & -14 & 3.269 & 6.302 & 1.928 \\
\hline 7 & P41982 & Superoxide dismutase & 4 & $\begin{array}{l}\text { EQGHLQIAAC } \\
\text { GELLEAIKR } \\
\text { HHAAYVNNLNATEEK } \\
\text { NVRPDYLK }\end{array}$ & $\begin{array}{l}2 \\
2 \\
2 \\
2\end{array}$ & -23.7 & 2.300 & 5.823 & 2.532 \\
\hline 8 & P68136 & $\alpha$-Actin & 3 & $\begin{array}{l}\text { DSYVGDEAQSK } \\
\text { QEYDEAGPSIVHR } \\
\text { AGFAGDDAPR }\end{array}$ & $\begin{array}{l}2 \\
2 \\
2\end{array}$ & -23.5 & 3.041 & 5.574 & 1.824 \\
\hline 9 & P13805 & Troponin T slow & 3 & $\begin{array}{l}\text { VDFDDIHR } \\
\text { DFDDIHR } \\
\text { PVVPP }\end{array}$ & $\begin{array}{l}1 \\
1 \\
1\end{array}$ & -16.3 & 1.899 & 5.534 & 2.914 \\
\hline
\end{tabular}


Table 1. Continued

\begin{tabular}{|c|c|c|c|c|c|c|c|c|c|}
\hline $\begin{array}{l}\text { Spot } \\
\text { no. }\end{array}$ & $\begin{array}{l}\text { Accession } \\
\text { no. }\end{array}$ & Protein & $\begin{array}{l}\text { Peptides } \\
\text { matched }\end{array}$ & Peptide sequence & Charge & Score & $\begin{array}{l}0-14 d \\
\text { CLFS }\end{array}$ & $\begin{array}{l}0-60 \mathrm{~d} \\
\text { CLFS }\end{array}$ & $\begin{array}{l}14 \mathrm{~d}-60 \mathrm{~d} \\
\text { CLFS }\end{array}$ \\
\hline 10 & P02563 & MHC slow/cardiac & 6 & $\begin{array}{l}\text { QAEEAEEOANTNLSK } \\
\text { VQLLHSONTSLINOK } \\
\text { VQHELDEAEER } \\
\text { KVOHELDEAEER } \\
\text { AVVEOTER } \\
\text { VRELENELEAEOK }\end{array}$ & $\begin{array}{l}1 \\
1 \\
2 \\
1 \\
1 \\
1\end{array}$ & -66.5 & 2.583 & 3.905 & 1.512 \\
\hline 11 & 014558 & $\alpha$ Crystallin-related HSP-B6 & 2 & $\begin{array}{l}\text { HFSPEEIAVK } \\
\text { MEIPVPVQPS }\end{array}$ & $\begin{array}{l}2 \\
1\end{array}$ & -10 & 1.858 & 3.541 & 1.906 \\
\hline 12 & P02645 & Troponin I slow & 1 & GLSLSALODLCR & 2 & -5.6 & 1.468 & 3.262 & 2.222 \\
\hline 13 & P41316 & $\alpha \mathrm{B}$-crystallin & 3 & $\begin{array}{l}\text { EEKPAVTAAPK } \\
\text { VLGDVIEVHGK } \\
\text { HFSPEELK }\end{array}$ & $\begin{array}{l}2 \\
2 \\
2\end{array}$ & -18.1 & 2.291 & 3.200 & 1.396 \\
\hline 14 & P19234 & $\begin{array}{l}\text { NADH-ubiquinone oxidore- } \\
\text { ductase }\end{array}$ & 3 & $\begin{array}{l}\text { AAAVLPVLDLAQR } \\
\text { VAEVLOVPPMR } \\
\text { GPGFGVQAGL }\end{array}$ & $\begin{array}{l}2 \\
2 \\
1\end{array}$ & -22.2 & 2.410 & 3.117 & 1.294 \\
\hline 15 & P04792 & HSP-27 & 4 & $\begin{array}{l}\text { TKDGVVEITGK } \\
\text { QLSSGVSEIR } \\
\text { QDEHGYISR } \\
\text { DGVVEITGK }\end{array}$ & $\begin{array}{l}2 \\
1 \\
2 \\
1\end{array}$ & -36.5 & 1.799 & 3.062 & 1.702 \\
\hline 16 & P30042 & ES1 Protein homologue & 3 & $\begin{array}{l}\text { GVEVTVGHEOEEGGK } \\
\text { NLSTFAVDGK } \\
\text { STFAVDGK }\end{array}$ & $\begin{array}{l}2 \\
1 \\
1\end{array}$ & -18.4 & 2.082 & 2.943 & 1.413 \\
\hline 17 & P21796 & $\begin{array}{l}\text { Voltage-dependent anion } \\
\text { channel } 1\end{array}$ & 3 & $\begin{array}{l}\text { LTFDSSFSPNTGK } \\
\text { YOIDPDACFSAK } \\
\text { VTOSNFAVGYK }\end{array}$ & $\begin{array}{l}2 \\
2 \\
2\end{array}$ & -27.1 & 1.634 & 2.650 & 1.622 \\
\hline 18 & P02170 & Myoglobin & 12 & $\begin{array}{l}\text { VEADLAGHGQEVLIR } \\
\text { HPGDFGADAO } \\
\text { VEADLAGHGO } \\
\text { HPGDFGADAQAAMSK } \\
\text { LAGHGOEVLIR } \\
\text { VEADLAGHGQEV } \\
\text { LFHTHPETLEK } \\
\text { LFHTHPETL } \\
\text { GHHEAEIKPL } \\
\text { HPGDFGADAQA } \\
\text { NDIAAOYK } \\
\text { GHHEAEIKPLAO }\end{array}$ & $\begin{array}{l}2 \\
2 \\
2 \\
2 \\
2 \\
2 \\
2 \\
2 \\
2 \\
2 \\
1 \\
2\end{array}$ & -117.5 & 1.673 & 2.518 & 1.505 \\
\hline 19 & P11177 & Pyruvate dehydrogenase & 4 & $\begin{array}{l}\text { GPNGASAGVAAQHSO } \\
\text { VTGADVPMPY } \\
\text { DFLIPIGK } \\
\text { VTGADVPMPYAK }\end{array}$ & $\begin{array}{l}2 \\
1 \\
1 \\
2\end{array}$ & -27.4 & 3.685 & 2.511 & 1.083 \\
\hline 20 & Q99LC5 & $\begin{array}{l}\text { Electron-transferring } \\
\text { flavoprotein }\end{array}$ & 2 & $\begin{array}{l}\text { TIYAGNALCTVK } \\
\text { OSTLVIAEH }\end{array}$ & $\begin{array}{l}2 \\
1\end{array}$ & -13.1 & 2.319 & 2.511 & 1.083 \\
\hline 21 & P49065 & Serum albumin & 3 & $\begin{array}{l}\text { ACVADESAANCDK } \\
\text { AILTECCEAADK } \\
\text { PEADVLCK }\end{array}$ & $\begin{array}{l}2 \\
2 \\
2\end{array}$ & -28.3 & 1.820 & 2.242 & 1.232 \\
\hline 22 & $09 C Z 13$ & $\begin{array}{l}\text { Ubiquinol-Cytochrome- } c \\
\text { reductase }\end{array}$ & 10 & $\begin{array}{l}\text { MVLAAAGGVEHQ } \\
\text { OGTPLAQAVEGPSENVR } \\
\text { AQAVEGPSENVR } \\
\text { LCTSATESEVTR } \\
\text { CTSATESEVTR } \\
\text { LDGTTPVCEDIGR }\end{array}$ & $\begin{array}{l}2 \\
2 \\
2 \\
2 \\
2 \\
2\end{array}$ & -104.5 & 1.234 & 2.025 & 1.641 \\
\hline
\end{tabular}


Table 1. Continued

\begin{tabular}{|c|c|c|c|c|c|c|c|c|c|}
\hline $\begin{array}{l}\text { Spot } \\
\text { no. }\end{array}$ & $\begin{array}{l}\text { Accession } \\
\text { no. }\end{array}$ & Protein & $\begin{array}{l}\text { Peptides } \\
\text { matched }\end{array}$ & Peptide sequence & Charge & Score & $\begin{array}{l}0-14 d \\
\text { CLFS }\end{array}$ & $\begin{array}{l}0-60 d \\
\text { CLFS }\end{array}$ & $\begin{array}{l}14 d-60 d \\
\text { CLFS }\end{array}$ \\
\hline & & & & $\begin{array}{l}\text { TSATESEVTR } \\
\text { IPLAEWESR } \\
\text { AQAVEGPSEN } \\
\text { RIPLAEWESR }\end{array}$ & $\begin{array}{l}2 \\
2 \\
2 \\
2\end{array}$ & & & & \\
\hline 23 & P11974 & $\begin{array}{l}\text { Pyruvate kinase, muscle } \\
\text { isoform }\end{array}$ & 4 & $\begin{array}{l}\text { LDIDSAPITAR } \\
\text { GSGTAEVELKK } \\
\text { PVAVALDTK } \\
\text { GSGTAEVELK }\end{array}$ & $\begin{array}{l}2 \\
2 \\
1 \\
2\end{array}$ & -24.9 & 1.975 & 2.005 & 1.105 \\
\hline 24 & P25705 & $\begin{array}{l}\text { ATP synthase, } \\
\text { mitochondrial F1 complex }\end{array}$ & 2 & $\begin{array}{l}\text { TIEDLNEAFPETK } \\
\text { AGLVDDFEK }\end{array}$ & $\begin{array}{l}2 \\
2\end{array}$ & -8.9 & 1.920 & 1.682 & -1.138 \\
\hline 25 & P81605 & Proteolysis-inducing factor & 3 & $\begin{array}{l}\text { ENAGEDPGLAR } \\
\text { QKENAGEDPGLA } \\
\text { KENAGEDPGLA }\end{array}$ & $\begin{array}{l}2 \\
2 \\
2\end{array}$ & -16.1 & 2.164 & 1.672 & 1.294 \\
\hline 26 & Q9GZV1 & $\begin{array}{l}\text { Ankyrin, skeletal muscle } \\
\text { isoform }\end{array}$ & 3 & $\begin{array}{l}\text { FLADGGSADTCDQFR } \\
\text { MDGTMEDSEAVQR } \\
\text { EIIDVGGION }\end{array}$ & $\begin{array}{l}2 \\
2 \\
1\end{array}$ & -17.1 & 1.308 & 1.670 & 2.183 \\
\hline 27 & P61604 & HSP-10 (chaperonin 10) & 1 & VLLPEYGGTK & 1 & -8.2 & 2.222 & 1.627 & -1.366 \\
\hline 28 & P04792 & HSP-27 & 4 & $\begin{array}{l}\text { TKDGVVEITGK } \\
\text { OLSSGVSEIR } \\
\text { DEHGYISR } \\
\text { ODEHGYISR } \\
\text { DGVVEITGKHEER } \\
\text { DGVVEITGK } \\
\text { SSGVSEIR }\end{array}$ & $\begin{array}{l}2 \\
1 \\
2 \\
2 \\
2 \\
1 \\
1\end{array}$ & -26.6 & 1.129 & 1.412 & 1.251 \\
\hline 29 & 001995 & Transgelin & 2 & $\begin{array}{l}\text { KYDEELEER } \\
\text { YDEELEER }\end{array}$ & $\begin{array}{l}2 \\
2\end{array}$ & -10.7 & $\begin{array}{l}\text { Not } \\
\text { detectable }\end{array}$ & $\begin{array}{l}\text { Not } \\
\text { detectable }\end{array}$ & $\begin{array}{l}\text { Positive } \\
\text { spot at } 60 d\end{array}$ \\
\hline 30 & P62804 & Histone-4 & 2 & $\begin{array}{l}\text { ISGLIYEETR } \\
\text { ISGLIYEETR }\end{array}$ & $\begin{array}{l}2 \\
2\end{array}$ & -12.2 & -1.266 & -1.371 & -1.083 \\
\hline 31 & P02602 & $\begin{array}{l}\text { Myosin light chain } \\
\text { MLC } 1 \text { fast }\end{array}$ & 7 & $\begin{array}{l}\text { DQGTYEDFVEGLR } \\
\text { VLGNPSNEEMNAK } \\
\text { ALGTNPTNAEVK } \\
\text { ALGTNPTNAEVKK } \\
\text { ITLSQVGDVLR } \\
\text { KVLGNPSNEEMNAK } \\
\text { EAFLLYDR } \\
\text { HVLATLGEK } \\
\text { VLGNPSN } \\
\text { ALGTNPTN } \\
\text { VFDKEGNGTVMGAELR } \\
\text { GTNPTNAEVKK } \\
\text { EGNGTVMGAELR }\end{array}$ & $\begin{array}{l}2 \\
2 \\
2 \\
2 \\
2 \\
2 \\
2 \\
2 \\
1 \\
1 \\
2 \\
2 \\
2\end{array}$ & -49.5 & -1.078 & -1.378 & -1.278 \\
\hline 32 & P30086 & $\begin{array}{l}\text { Phosphatidylethanolamine } \\
\text { binding protein }\end{array}$ & 3 & $\begin{array}{l}\text { LYTLVLTDPDAPSR } \\
\text { TLVLTDPDAPSR } \\
\text { LYEOLSGK }\end{array}$ & $\begin{array}{l}2 \\
2 \\
1\end{array}$ & -20.8 & 1.062 & -2.046 & -2.173 \\
\hline 33 & P00939 & Triosephosphate isomerase & 17 & $\begin{array}{l}\text { HVFGESDELIGQK } \\
\text { SNVSDAVAOSTR } \\
\text { IIYGGSVTGATCK } \\
\text { TATPQOAOEVHEK } \\
\text { DCGATWVVLGH } \\
\text { DCGATWVVLGHSER } \\
\text { ELASQPDVDGF } \\
\text { TPQOAOEVHEK } \\
\text { VAHALSEGLGVIAC }\end{array}$ & $\begin{array}{l}2 \\
2 \\
2 \\
2 \\
2 \\
2 \\
2 \\
2 \\
2\end{array}$ & -183.9 & 1.076 & -2.052 & -2.207 \\
\hline
\end{tabular}


Table 1. Continued

\begin{tabular}{|c|c|c|c|c|c|c|c|c|c|}
\hline $\begin{array}{l}\text { Spot } \\
\text { no. }\end{array}$ & $\begin{array}{l}\text { Accession } \\
\text { no. }\end{array}$ & Protein & $\begin{array}{l}\text { Peptides } \\
\text { matched }\end{array}$ & Peptide sequence & Charge & Score & $\begin{array}{l}0-14 d \\
\text { CLFS }\end{array}$ & $\begin{array}{l}0-60 d \\
\text { CLFS }\end{array}$ & $\begin{array}{l}14 \mathrm{~d}-60 \mathrm{~d} \\
\text { CLFS }\end{array}$ \\
\hline & & & & $\begin{array}{l}\text { IAVAAQNCYK } \\
\text { QAOEVHEK } \\
\text { VPADTEVVCAPPTA } \\
\text { VTNGAFTGEISPGMIK } \\
\text { TGEISPGMIK } \\
\text { VVLAY } \\
\text { IIYGG } \\
\text { YGGSVTGATCK }\end{array}$ & $\begin{array}{l}2 \\
2 \\
2 \\
2 \\
2 \\
2 \\
1 \\
1 \\
1\end{array}$ & & & & \\
\hline 34 & P00558 & Phosphoglycerate kinase 1 & 4 & $\begin{array}{l}\text { FHVEEEGK } \\
\text { DCVGPEVEK } \\
\text { ITLPVDFVTADK } \\
\text { ALMDEVVK }\end{array}$ & $\begin{array}{l}2 \\
2 \\
2 \\
2\end{array}$ & -20.8 & -1.280 & -2.718 & -2.214 \\
\hline 35 & P02624 & Parvalbumin & 9 & $\begin{array}{l}\text { TLMAAGDKDGDGK } \\
\text { AAAESFDHK } \\
\text { MAAGDKDGDGK } \\
\text { VFHILDKDK } \\
\text { AAGDKDGDGK } \\
\text { IGADEFSTLVSES } \\
\text { AIGAF } \\
\text { TLMAAGDK } \\
\text { AGDKDGDGK }\end{array}$ & $\begin{array}{l}2 \\
2 \\
2 \\
2 \\
2 \\
2 \\
1 \\
1 \\
1\end{array}$ & -74.8 & -2.184 & -3.493 & -1.599 \\
\hline 36 & P02643 & Troponin I fast & 2 & $\begin{array}{l}\text { SSKELEDMNOK } \\
\text { MSADAMLK }\end{array}$ & $\begin{array}{l}2 \\
1\end{array}$ & -8.3 & -1.140 & -4.387 & -3.848 \\
\hline 37 & P14152 & Malate dehydrogenase & 3 & $\begin{array}{l}\text { GEFITTVQQR } \\
\text { KLSSAMSAAK } \\
\text { LGVTADDVK }\end{array}$ & $\begin{array}{l}2 \\
2 \\
1\end{array}$ & -17.2 & 1.050 & -4.480 & -4.705 \\
\hline 38 & P08507 & $\begin{array}{l}\text { Glycerol-3-phosphate } \\
\text { dehydrogenase }\end{array}$ & 9 & $\begin{array}{l}\text { IVGGNAAQLAQFDPR } \\
\text { LPPNVVAVPDVVK } \\
\text { ELHSILOHK } \\
\text { FCETTIGCK } \\
\text { CLONHPEHM } \\
\text { DQAOGOLLK } \\
\text { OLMOTPNFR } \\
\text { IASEVADEK } \\
\text { ANAIGISLIK }\end{array}$ & $\begin{array}{l}2 \\
2 \\
2 \\
2 \\
2 \\
2 \\
1 \\
2 \\
2 \\
2\end{array}$ & -77.9 & -1.264 & -4.712 & -3.727 \\
\hline 39 & P15122 & Aldose reductase & 3 & $\begin{array}{l}\text { REELFIVSK } \\
\text { SPPGQVTEAVK } \\
\text { EELFIVSK }\end{array}$ & $\begin{array}{l}2 \\
2 \\
1\end{array}$ & -17.7 & -1.264 & -4.712 & -3.727 \\
\hline 40 & P02608 & Myosin light chain MLC 2 & 8 & $\begin{array}{l}\text { GADPEDVITGAFK } \\
\text { NICYVITHGDAK } \\
\text { PEDVITGAFK } \\
\text { EAFTVIDQNR } \\
\text { NEELDAMMK } \\
\text { ICYVITHGDAK } \\
\text { DOTOIOEFK } \\
\text { AFPPDVGGNVDYK }\end{array}$ & $\begin{array}{l}2 \\
2 \\
2 \\
2 \\
2 \\
2 \\
2 \\
2\end{array}$ & -69.9 & -1.795 & -5.690 & -3.170 \\
\hline 41 & P02603 & $\begin{array}{l}\text { Myosin light chain } \\
\text { MLC } 3 \text { fast }\end{array}$ & 15 & $\begin{array}{l}\text { DQGTYEDFVEGLR } \\
\text { VLGNPSNEEMNAK } \\
\text { ALGTNPTNAEVK } \\
\text { ALGTNPTNAEVKK } \\
\text { SFSADOIAEFK } \\
\text { ITLSOVGDVLR }\end{array}$ & $\begin{array}{l}2 \\
2 \\
2 \\
2 \\
2 \\
2\end{array}$ & -151.9 & -3.643 & -13.208 & -3.626 \\
\hline
\end{tabular}


Table 1. Continued

\begin{tabular}{|c|c|c|c|c|c|c|c|c|c|}
\hline $\begin{array}{l}\text { Spot } \\
\text { no. }\end{array}$ & $\begin{array}{l}\text { Accession } \\
\text { no. }\end{array}$ & Protein & $\begin{array}{l}\text { Peptides } \\
\text { matched }\end{array}$ & Peptide sequence & Charge & Score & $\begin{array}{l}0-14 d \\
\text { CLFS }\end{array}$ & $\begin{array}{l}0-60 d \\
\text { CLFS }\end{array}$ & $\begin{array}{l}14 d-60 d \\
\text { CLFS }\end{array}$ \\
\hline & & & & KVLGNPSNEEMNAK & 2 & & & & \\
\hline & & & & SADQIAEFK & 2 & & & & \\
\hline & & & & EAFLLYDR & 2 & & & & \\
\hline & & & & HVLATLGEK & 2 & & & & \\
\hline & & & & VLGNPSN & 1 & & & & \\
\hline & & & & ALGTNPTN & 1 & & & & \\
\hline & & & & VFDKEGNGTVMGAELR & 2 & & & & \\
\hline & & & & GTNPTNAEVKK & 2 & & & & \\
\hline & & & & EGNGTVMGAELR & 2 & & & & \\
\hline
\end{tabular}

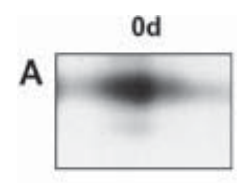

$14 d$

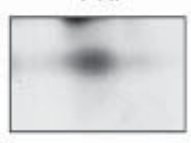

B
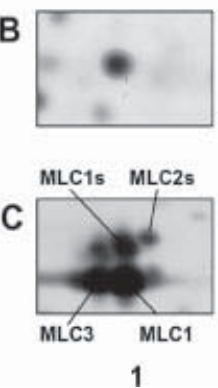
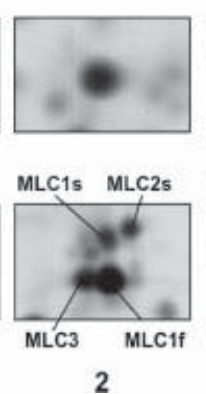

$60 d$
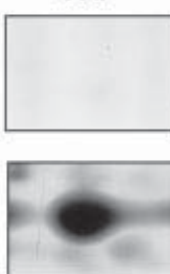

$4 \mathrm{TnT}$, slow

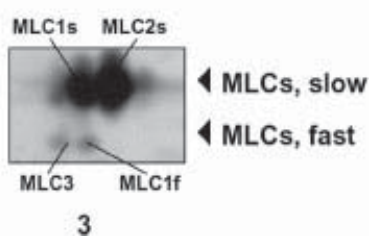

Figure 2. 2-D gel electrophoretic analysis of stimulation-induced changes in key contractile protein isoforms. Shown is an expanded view of silver-stained landmark 2-D protein spots, depicted in their original position in the representative gels of Fig. 1. Lanes 1 to 3 show gels of unstimulated $(0 d), 14$-day stimulated (14d) and 60-day stimulated (60d) fast muscles, respectively. The gradual change in the expression levels of fast Troponin-I ( $A_{i}$ Tnl), slow Troponin-T (B; TnT) and the various fast and slow myosin light chains (C; MLCs) clearly demonstrated that the chronic low-frequency stimulation of the peroneal nerve induced a fast-toslow fibre transition process in the tibialis anterior muscle.

\subsection{Identification of protein species with a changed expression pattern in stimulated fast muscle}

Taking these above described isoform switching of troponin and myosin light chain as standards for the dynamic process of fibre transformation, we then determined the overall changes in the accessible fast muscle proteome after 2 and 8.5 wk of conditioning. Figure 3 illustrates the master 2-D reference gel of chronic low-frequency stimulated fast muscle. Shown is a silver-stained gel of 60-day stimulated fast muscle. Muscle proteins with a drastically different expression level, as determined by fluorescent DIGE analysis (Figs. 1D and F), are marked by circles and are listed in Table 1. Following chronic electrostimulation, a differential expression pattern was observed for 41 protein species with 29 increased and 12 decreased muscle proteins. Identified classes of proteins that change during the fast-to-slow transition process belong to the contractile machinery, ion homeostasis, vascularization, metabolism and stress. Increased protein species include the oxygen transporters hemoglobin and myoglobin, slow-twitch isoforms of contractile elements such as myosin light chain, MHC, actin, troponin I and troponin $\mathrm{T}$, the fatty acid transporters albumin and FABP-3, mitochondrial proteins including Cytochrome-c oxidase, NADH-ubiquinone oxidoreductase, F1 complex ATP synthase, proteolysis-inducing factor, pyruvate dehydrogenase, ES1 protein homologue and electron-transferring flavoprotein, stress proteins such as HSP-27, $\alpha$-crystallin-related HSP-B6, $\alpha$ B-crystalline and HSP-10, the anion-channel VDAC-1, the enzymes pyruvate kinase and superoxide dismutase, as well as ankyrin, cofilin-2 and transgelin. The fold-change in transgelin expression could not be determined due to an obstructing troponin-containing spot in 0- and 14-day stimulated samples (not shown). However, a distinct 2-D spot representing transgelin was identified in 60-day stimulated specimens. See below for the immunoblot analysis of transgelin in transforming fibres. In contrast to the large number of increased muscle protein species following chronic electrostimulation, a reduced expression was shown for fast isoforms of the MHC and myosin light chain, the enzyme aldose reductase and the cytosolic malate dehydrogenase, the cytosolic $\mathrm{Ca}^{2+}$-binding protein parvalbumin, histone-4, the binding protein of the glycerophospholipid phosphotidylethanolamine, and key glycolytic enzymes such as aldolase, triosephosphate isomerase, phosphoglycerate kinase and glycerol-3-phosphate dehydrogenase.

Proteins of interest, that had been identified by DIGE analysis to exhibit a significant change in their expression following chronic low-frequency stimulation, were picked from preparative silver-stained 2-D gels and subsequently identified by ESI-MS/MS. Figure 4 shows a representative overview of transformation biomarkers with expanded views 


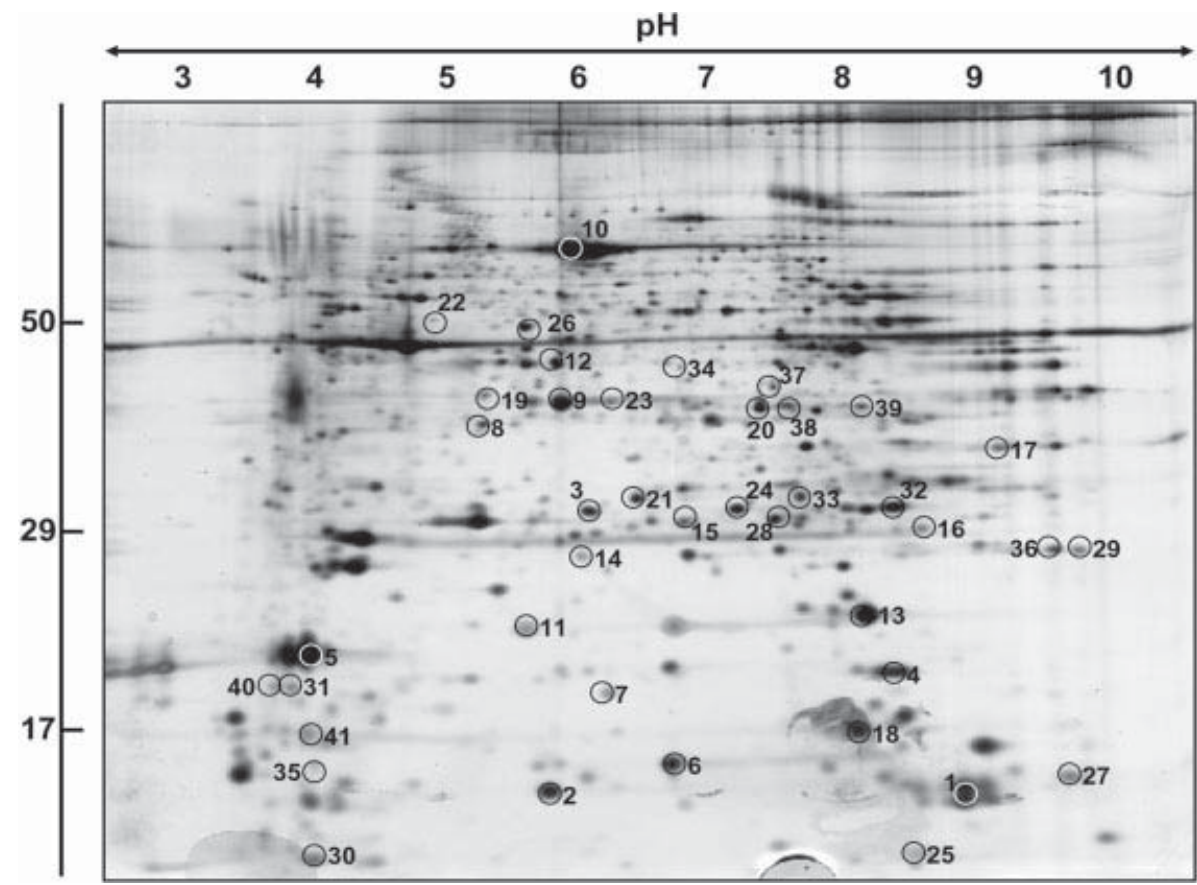

Figure 3. Master 2-D reference gel of chronic low-frequency stimulated fast muscle. Shown is a silver-stained 2-D gel of 60-day stimulated fast muscle. IEF has been carried out on $24 \mathrm{~cm} \mathrm{pH} \mathrm{3-}$ 10 IPG strips in the first dimension and standard $12 \%$ polyarcylamide slab gels in the second dimension. The $\mathrm{pH}$-values of the first dimension gel system and molecular mass standards (in $\mathrm{kDa}$ ) of the second dimension are indicated on the top and on the left of the panels, respectively. Muscle proteins with a drastically different expression level, as determined by fluorescent DIGE analysis (Fig. 1), are marked by circles and are numbered 1-41. See Table 1 for a detailed listing of skeletal muscle proteins with a changed abundance in chronic low-frequency stimulated fast muscle.

of the 2-D spot pattern of specific muscle proteins. Changes in individual 2-D spots demonstrated the differential effect of chronic electrostimulation on the various classes of muscle proteins. The observed drastic increase of the slow MHC isoform at 14 and 60 days of stimulation (Fig. 4A) was in stark contrast to the transient increase in the cytosolic $\mathrm{Ca}^{2+}$-binding protein parvalbumin and the oxygen-carrier myoglobin after 2 wk of stimulation followed by a decrease at day 60 of muscle conditioning (Figs. 4B and C). A continuous increase in the abundance of haemoglobin, the cardiac fatty acid binding protein FABP-3, Cytochrome $c$, the heat shock proteins $\alpha \mathrm{B}$-crystallin and HSP- $\beta 6$, and the actin binding protein cofilin-2 were revealed by DIGE analysis (Figs. 4D-I). Since the relatively low-abundance protein cofilin-2 had not previously been implicated to play a role in muscle transformation, this protein may represent potentially a new biomarker of the fast-to-slow transition process. This finding clearly demonstrates the superior sensitivity of DIGE analysis as compared to conventional proteomic approaches employing silver or commassie staining. Representative 2-D spots of transgelin, another newly identified potential biomarker of fibre shifting, are not shown due to the fact that this protein spot was covered in electrophoretically separated 0 - and 14-day stimulated samples by a 2-D spot representing fast troponin. It was therefore not possible to determine the exact degree of transgelin increase following chronic low-frequency stimulation, but the protein was clearly identified in 60-day stimulated fibres. In order to determine the fate of transgelin during the fast-toslow transition process, immunoblotting was employed as described below.

\subsection{Immunoblot analysis of fast-to-slow transition process following chronic electrostimulation}

To confirm the results from our DIGE analysis, changes in the abundance of potential new biomarkers of muscle transformation were investigated by immunoblot analysis. A select group of proteins, which were reliably recognized by antibody labelling, were studied in 0-, 14-, and 60-day chronic electrostimulated specimens. To demonstrate a successful fast-to-slow transition, and in order to provide internal standards of established alterations in known muscle proteins [4], the immunodecoration of the fast and slow isoforms of the sarcoplasmic reticulum $\mathrm{Ca}^{2+}$-ATPase and the MHC were carried out. As illustrated in Figs. 5A-D, a drastic increase in the slow isoforms SERCA2 and $\mathrm{MHC}_{\mathrm{s}}$ were observed and a concomitant decreased abundance of their fast counterparts, SERCA1 and $\mathrm{MHC}_{\mathrm{f}}$. Although antibodies to fast MHC do not differentiate between the various isoforms of this contractile protein, the gradual replacement of MHCs from the MHCII isoforms to MHCI has previously been demonstrated by detailed gel electrophoretic analyses as reviewed by Pette $e t a l$. $[4,48]$. Thus, the immunoblotting survey shown here confirmed the previously described alterations in $\mathrm{Ca}^{2+}$-uptake units of the sarcoplasmic reticulum during muscle conditioning and the modified myosin isoform expression pattern in fibres with slower switch characteristics, and thereby the usefulness of employing 1-D immunoblotting to determine changes in the expression of muscle marker proteins. The visualization of antibody binding by ECL revealed a drastic increase in DIGE-determined biomarkers of fibre type shifting, such as the muscle-specific cofilin-2 protein, 


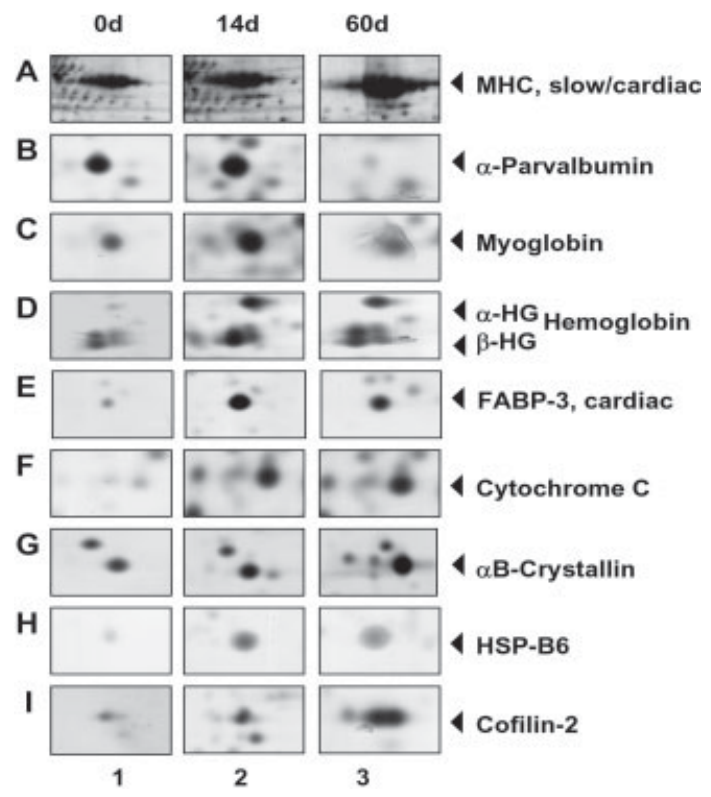

Figure 4. Differential expression pattern of skeletal muscle proteins following chronic low-frequency stimulation. Shown is an expanded view of silver-stained 2-D protein spots, depicted in their original position in the representative gels of Fig. 1, which show a significant change in abundance as judged by DIGE analysis. Lanes 1 to 3 show gels of unstimulated (Od), 14-day stimulated (14d) and 60-day stimulated (60d) fast muscles, respectively. Panels A to I illustrate the gradual change in the expression levels of muscle markers $\mathrm{MHC}$, parvalbumin, myoglobin, hemoglobin, cardiac fatty acid binding protein (FABP-3), Cytochrome $c, \alpha \mathrm{B}$ crystallin, heat shock protein HSP-B6 and cofilin-2.

the heat shock protein $\alpha \mathrm{B}$-crystallin, transgelin and the cardiac fatty acid binding-protein FABP-3 (Figs. 5E-H). The immunoblot analysis clearly agreed with the results from the DIGE screening of the muscle transition process. It confirmed the dramatic increase in the expression of new markers of the fast-to-slow transformation process, such as cofilin-2 and transgelin, which had not previously been described by conventional biochemical studies.

\section{Discussion}

Fibre type shifting plays an important role during development, mature muscle adaptation processes, ageing and many neuromuscular diseases [1-4]. It is therefore essential to identify reliable skeletal muscle protein markers of fast-toslow muscle transformation, which might be helpful in establishing new molecular indicators of physiological and pathological fibre alterations. Building on previously established 2D skeletal muscle reference maps [10, 11], we carried out a comprehensive proteomic profiling of the chronic lowfrequency stimulated rabbit tibialis anterior muscle and determined changes in the expression pattern of key muscle protein species. DIGE analysis and ESI-MS/MS for protein 0d 14d 60d

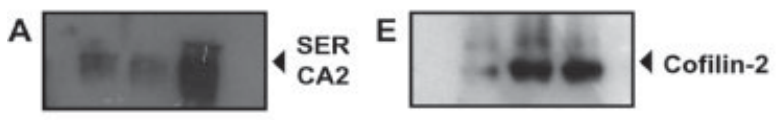

B

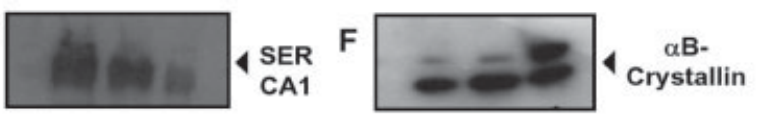

C

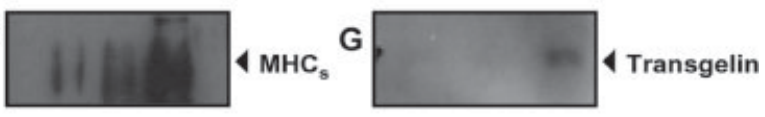

D

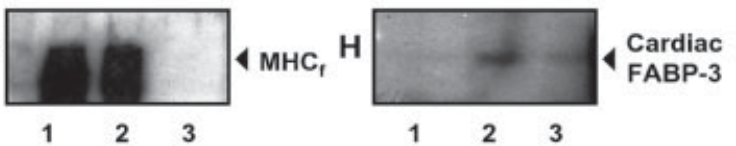

Figure 5. Immunoblot analysis of key skeletal muscle proteins following chronic low-frequency stimulation. Shown are identical immunoblots labelled with antibodies to the slow SERCA2 isoform of the sarcoplasmic reticulum $\mathrm{Ca}^{2+}$-ATPase (A), the fast SERCA1 isoform of the sarcoplasmic reticulum $\mathrm{Ca}^{2+}$-ATPase (B), the slow $\mathrm{MHC}_{\mathrm{s}}$ isoform of the $\mathrm{MHC}(\mathrm{C})$, the fast $\mathrm{MHC}_{\mathrm{f}}$ isoform of the $\mathrm{MHC}(\mathrm{D})$, cofilin-2 (E), $\alpha \mathrm{B}$-crystallin (F), transgelin (G), and the cardiac fatty acid binding protein FABP-3 $(\mathrm{H})$. Lanes 1 to 3 represent muscle preparations from 0 -, 14- and 60-day low-frequency stimulated tibialis anterior muscle, respectively.

identification and immunoblotting clearly confirmed established changes in representative protein species from distinct functional muscle protein groupings, and uncovered interesting new transformation biomarkers. This included a drastic stimulation-induced increase in the abundance of the actin binding protein cofilin- 2 and the cytoskeletal protein transgelin. Thus, besides confirming the findings from numerous physiological and biochemical studies on muscle conditioning over the past decades $[2,4,41]$ and extending the preliminary results from a nonfluorescent proteomics study [35], this report clearly shows the usefulness of employing high-resolution 2-DE and ESI-MS/MS for identifying new skeletal muscle biomarkers.

Changes in nutritional supply, fluctuations in hormonal factors, alterations in neuromuscular activity and modifications in mechanical loading conditions may all affect skeletal muscle plasticity [4]. Consequently, resistance training, endurance training, muscle immobilization and zero-gravity conditions may trigger distinct adjustments in the skeletal muscle proteome $[1,3,59]$. In addition, fibre type shifting or major alterations in muscle isoform expression levels also occur in various pathophysiological situations, such as traumatic denervation, disuse atrophy and sarcopenia of old age [60-62]. The DIGE analysis presented here supports the idea that the fast-to-slow transition process includes distinct changes in the muscle protein complement that is involved in the contractile machinery, ion homeostasis, excitationcontraction coupling, capillarization, metabolism, stress response and cytoskeletal support. The observed alterations in the expression of cofilin-2, transgelin, hemoglobin, cardiac 
fatty acid binding protein FABP-3, albumin, $\alpha$ B-crystallin, HSP-27 and myoglobin are especially interesting findings for a better understanding of the transformation process. A chronic increase in contractile activity and a shift to oxidative muscle metabolism would require a higher demand in oxygen and fuel supply in the form of fatty acids. Consequently the drastic increase in the oxygen-transporter myoglobin, the intracellular fatty acid binding protein FABP-3 and the extracellular fatty acid transporter albumin strongly indicates that these three central metabolic regulators could function as limiting factors of oxidative metabolism in fast fibres [40, 63]. The findings from our DIGE analysis clearly establish the small cytosolic FABP-3 protein as a suitable biomarker of muscle fibre type shifting. It is, however, difficult to make proper conclusions about the changed levels in the blood protein hemoglobin, which increased concentration might be due to varying amounts of this oxygen carrier being trapped in muscle tissue.

In agreement with the stimulation-induced change in the contractile apparatus towards slower isoforms, the decrease in glycolytic proteins and the increase in mitochondrial enzymes, an increase in markers of capillarization could be expected [64]. This report shows for the first time that the cytoskeletal protein transgelin [65] exists in longterm stimulated skeletal muscles. Transgelin, also referred to as SM22 $\alpha$ [66], has been localized to vascular smooth muscle cells throughout the arterial and venous system. It has been described in visceral smooth muscle cells in various organs and the heart, but as being absent from mature skeletal muscle [66]. The electrostimulation-dependent induction revealed by DIGE analysis suggests transgelin as another excellent biomarker of fast-to-slow fibre transition. The same is true for the actin binding protein cofilin-2, which plays a critical role in actin filament dynamics [67]. The muscle-specific cofilin isoform is believed to be important for the growth of myofibrils in developing and regenerating muscle cells $[68,69]$. In analogy, cofilin-2 up-regulation in transforming fibres could also be a response to remodeling of the contractile apparatus towards slower twitch properties.

Interestingly, during muscle transformation, identifiable heat shock proteins were found to be exclusively increased in their abundance, indicating that fibre modifications probably depend heavily on chaperone function. As previously discussed by Neufer and Benjamin [70], $\alpha$ B-crystallin expression may be directly regulated by the demand for oxidative metabolism. Besides $\alpha \mathrm{B}$-crystallin, also its ancestral-related heat shock protein HSP-27 and chaperonin-10 were increased following chronic low-frequency stimulation. Previous studies by Maier et al. [71] have shown that degeneration-regeneration cycle occur during stimulation-induced changes. Besides transdifferentiation from fast to slow fibres, other processes seem to occur in parallel such as the degradation of the fastest glycolytic fibre population and the activation of the satellite cell pool for the creation of new fibres with slower twitch characteristics. In contrast to the fast-to-slow transformation mechanism, in severe muscle degeneration, as occurs in X-linked muscular dystrophy, there appears to be a differential expression pattern of key chaperones. A recent MS-based proteomics and immunoblotting survey revealed that HSP-20, GRP-75 and HSP-90 are reduced in dystrophin-deficient fibres. On the other hand, cvHSP and HSP110 were drastically increased in their expression in dystrophinopathy [26]. This suggests $\alpha \mathrm{B}$-crystallin as another suitable biomarker of fibre type shifting.

In conclusion, the proteomic profiling of the fast-to-slow fibre transition process reported here has clearly shown that skeletal muscles represent a tissue type with an extremely high degree of plasticity that can swiftly adapt to changed functional demands on the protein expression level. The previous generation of a detailed 2-D protein map of the soluble gastrocnemius proteome versus its soleus counterpart by Gelfi et al. [17] has established a high density of MLC-1f, fast troponin I, glycolytic enzymes, creatine kinase and adenylate kinase in gastrocnemius muscle and MLC1s, slow troponin $\mathrm{C}$, myoglobin and carbonic anhydrase 3 as slow-twitch markers present at high concentration in soleus fibres. In analogy, the DIGE analysis presented here demonstrated that the transformed tibialis anterior muscle exhibited high levels of MLC1s, MHCs, slow troponin I, myoglobin and low levels of MLC1f, fast troponin I and glycolytic enzymes. Hence, the conditioned fast muscle protein complement resembles that of a slow-twitching soleus muscle. This study has clearly established a more comprehensive picture of the overall fiber transition process than has been previously reported [4]. Modern proteomics technology presents itself as an ideal biochemical tool for the study of complex biological systems and processes, such as the finely tuned physiological adaptation of skeletal muscles following chronic low-frequency stimulation.

Research was supported by principal investigator grants from Science Foundation Ireland (SFI-04/RP1/B499 to M. J. D.; SFI-04/IN3/B614 to K. O.), a project grant from the Irish Health Research Board (HRB-RP03/2001) and a training and mobility grant from the European Commission (RTN2-200100337), as well as equipment grants from the Irish Health Research Board (HRB-EQ/2003/3, HRB-EQ/2004/2). The authors thank Dr. Dirk Pette, Professor emeritus at the University of Konstanz, for his generous support of our muscle plasticity project. The Irish Higher Education Authority and the Deutscher Akademischer Austauschdienst provided funds for laboratory visits of the Maynooth team in Germany.

\section{References}

[1] Fluck, M., Functional, structural and molecular plasticity of mammalian skeletal muscle in response to exercise stimuli. J. Exp. Biol. 2006, 209, 2239-2248.

[2] Hood, D. A., Irrcher, I., Ljubicic, V., Joseph, A. M., Coordination of metabolic plasticity in skeletal muscle. J. Exp. Biol. 2006, 209, 2265-2275. 
[3] Goldspink, G., Gene expression in muscle in response to exercise. J. Muscle Res. Cell. Motil. 2003, 24, 121-126.

[4] Pette, D., The adaptive potential of skeletal muscle fibers. Can. J. Appl. Physiol. 2002, 27, 423-448.

[5] Aebersold, R., Mann, M., Mass spectrometry-based proteomics. Nature 2003, 422, 198-207.

[6] de Hoog, C. L., Mann, M., Proteomics. Annu. Rev. Genomics Hum. Genet. 2004, 5, 267-293.

[7] McGregor, E., Dunn, M. J., Proteomics of the heart: Unraveling disease. Circ. Res. 2006, 98, 309-321.

[8] Westbrook, J. A., Wheeler, J. X., Wait, R., Welson, S. Y., Dunn, M. J., The human heart proteome: Two-dimensional maps using narrow-range immobilised $\mathrm{pH}$ gradients. Electrophoresis 2006, 27, 1547-1555.

[9] Isfort, R. J., Proteomic analysis of striated muscle. J. Chromatogr. B 2002, 771, 155-165.

[10] Yan, J. X., Harry, R. A., Wait, R., Welson, S. Y. et al., Separation and identification of rat skeletal muscle proteins using two-dimensional gel electrophoresis and mass spectrometry. Proteomics 2001, 1, 424-434.

[11] Sanchez, J. C., Chiappe, D., Converset, V., Hoogland, C. et al., The mouse SWISS-2D PAGE database: A tool for proteomics study of diabetes and obesity. Proteomics 2001, 1, 136-163.

[12] Gelfi, C., De Palma, S., Cerretelli, P., Begum, S., Wait, R., Two-dimensional protein map of human vastus lateralis muscle. Electrophoresis 2003, 24, 286-295.

[13] Bouley, J., Chambon, C., Picard, B., Mapping of bovine skeletal muscle proteins using two-dimensional gel electrophoresis and mass spectrometry. Proteomics 2004, 4, 1811-1824.

[14] Kjaersgard, I. V., Norrelykke, M. R., Jessen, F., Changes in cod muscle proteins during frozen storage revealed by proteome analysis and multivariate data analysis. Proteomics 2006, 6, 1606-1518.

[15] Kim, N. K., Joh, J. H., Park, H. R., Kim, O. H. et al., Differential expression profiling of the proteomes and their mRNAs in porcine white and red skeletal muscles. Proteomics 2004, 4, 3422-3428.

[16] Okumura, N., Hashida-Okumura, A., Kita, K., Matsubae, M. et al., Proteomic analysis of slow- and fast-twitch skeletal muscles. Proteomics 2005, 5, 2896-2906.

[17] Gelfi, C., Vigano, A., De Palma, S., Ripamonti, M. et al., 2-D protein maps of rat gastrocnemius and soleus muscles: A tool for muscle plasticity assessment. Proteomics 2006, 6, 321-340.

[18] Bihan, M. C., Hou, Y., Harris, N., Tarelli, E., Coulton, G. R., Proteomic analysis of fast and slow muscles from normal and kyphoscoliotic mice using protein arrays, 2-DE and MS. Proteomics 2006, 6, 4646-4661.

[19] Doherty, M. K., McLean, L., Hayter, J. R., Pratt, J. M. et al., The proteome of chicken skeletal muscle: Changes in soluble protein expression during growth in a layer strain. Proteomics 2004, 4, 2082-2093.

[20] Kislinger, T., Gramolini, A. O., Pan, Y., Rahman, K. et al., Proteome dynamics during $\mathrm{C} 2 \mathrm{C} 12$ myoblast differentiation. Mol. Cell. Proteomics 2005, 4, 887-901.

[21] Bouley, J., Meunier, B., Chambon, C., De Smet, S. et al., Proteomic analysis of bovine skeletal muscle hypertrophy. Proteomics 2005, 5, 490-500.
[22] Ge, Y., Molloy, M. P., Chamberlain, J. S., Andrews, P. C., Proteomic analysis of $\mathrm{mdx}$ skeletal muscle: Great reduction of adenylate kinase 1 expression and enzymatic activity. Proteomics 2003, 3, 1895-1903.

[23] Ge, Y., Molloy, M. P., Chamberlain, J. S., Andrews, P. C., Differential expression of the skeletal muscle proteome in $\mathrm{mdx}$ mice at different ages. Electrophoresis 2004, 25, 2576-2585.

[24] Doran, P., Dowling, P., Lohan, J., McDonnell, K. et al., Subproteomics analysis of $\mathrm{Ca}+$-binding proteins demonstrates decreased calsequestrin expression in dystrophic mouse skeletal muscle. Eur. J. Biochem. 2004, 271, 3943-3952.

[25] Doran, P., Dowling, P., Donoghue, P., Buffini, M., Ohlendieck, K., Reduced expression of regucalcin in young and aged mdx diaphragm indicates abnormal cytosolic calcium handling in dystrophin-deficient muscle. Biochim. Biophys. Acta. 2006, 1764, 773-785.

[26] Doran, P., Martin, G., Dowling, P., Jockusch, H., Ohlendieck, K., Proteome analysis of the dystrophin-deficient MDX diaphragm reveals a drastic increase in the heat shock protein cvHSP. Proteomics 2006, 6, 4610-4621.

[27] De Palma, S., Morandi, L., Mariani, E., Begum, S. et al., Proteomic investigation of the molecular pathophysiology of dysferlinopathy. Proteomics 2006, 6, 379-385.

[28] Isfort, R. J., Wang, F., Greis, K. D., Sun, Y. et al., Proteomic analysis of rat soleus and tibialis anterior muscle following immobilization. J. Chromatogr. B 2002, 769, 323-332.

[29] Isfort, R. J., Wang, F., Greis, K. D., Sun, Y. et al., Proteomic analysis of rat soleus muscle undergoing hindlimb suspension-induced atrophy and reweighting hypertrophy. Proteomics 2002, 2, 543-550.

[30] Cobon, G. S., Verrills, N., Papakostopoulos, P., Eastwood, H., Linnane, A. W., The proteomics of ageing. Biogerontology 2002, 133-136.

[31] Kanski, J., Hong, S. J., Schoneich, C., Proteomic analysis of protein nitration in aging skeletal muscle and identification of nitrotyrosine-containing sequences in vivo by nanoelectrospray ionization tandem mass spectrometry. J. Biol. Chem. 2005, 280, 24261-24266.

[32] Piec, I., Listrat, A., Alliot, J., Chambon, C. et al., Differential proteome analysis of aging in rat skeletal muscle. FASEB $\mathrm{J}$. 2005, 19, 1143-1145.

[33] Kanski, J., Schoneich, C., Protein nitration in biological aging: Proteomic and tandem mass spectrometric characterization of nitrated sites. Methods Enzymol. 2005, 396, 160-171.

[34] Gelfi, C., Vigano, A., Ripamonti, M., Pontoglio, A. et al., The human muscle proteome in aging. J. Proteome Res. 2006, 5, 1344-1353.

[35] Donoghue, P., Doran, P., Dowling, P., Ohlendieck, K., Differential expression of the fast skeletal muscle proteome following chronic low-frequency stimulation. Biochim. Biophys. Acta 2005, 1752, 166-176.

[36] De la Torre, J. I., Griffin, D. W., Vasconez, L. O., Harvesting the latissimus dorsi muscle for cardiomyoplasty. Plast. Reconstr. Surg. 2000, 105, 83-88.

[37] Ljubicic, V., Adhihetty, P. J., Hood, D. A., Application of animal models: Chronic electrical stimulation-induced contractile activity. Can. J. Appl. Physiol. 2005, 30, 625-643.

[38] Hicks, A., Ohlendieck, K., Gopel, S. O., Pette, D., Early functional and biochemical adaptations to low-frequency stimu- 
lation of rabbit fast-twitch muscle. Am. J. Physiol. 1997, 2273, C297-C305.

[39] Reichmann, H., Wasl, R., Simoneau, J. A., Pette, D., Enzyme activities of fatty acid oxidation and the respiratory chain in chronically stimulated fast-twitch muscle of the rabbit. Pflugers Arch. 1991, 418, 572-574.

[40] Kaufmann, M., Simoneau, J. A., Veerkamp, J. H., Pette, D., Electrostimulation-induced increases in fatty acid-binding protein and myoglobin in rat fast-twitch muscle and comparison with tissue levels in heart. FEBS Lett. 1989, 245, 181-184.

[41] Leeuw, T., Pette, D., Coordinate changes in the expression of troponin subunit and myosin heavy-chain isoforms during fast-to-slow transition of low-frequency-stimulated rabbit muscle. Eur. J. Biochem. 1993, 213, 1039-1046.

[42] Gonzalez, B., Negredo, R., Hernando, R., Manso, R., Protein variants of skeletal muscle regulatory myosin light chain isoforms: prevalence in mammals, generation and transitions during muscle remodelling. Pflugers Arch. 2002, 443, 377-386.

[43] Ohlendieck, K., Froemming, G. R., Murray, B. E., Maguire, P. B. et al., Effects of chronic low-frequency stimulation on $\mathrm{Ca} 2+$-regulatory membrane proteins in rabbit fast muscle. Pflugers Arch. 1999, 438, 700-708.

[44] Froemming, G. R., Murray, B. E., Harmon, S., Pette, D., Ohlendieck, K., Comparative analysis of the isoform expression pattern of $\mathrm{Ca}(2+)$-regulatory membrane proteins in fast-twitch, slow-twitch, cardiac, neonatal and chronic low-frequency stimulated muscle fibers. Biochim. Biophys. Acta 2000, 1466, 151-168.

[45] Sketelj, J., Crne-Finderle, N., Strukelj, B., Trontelj, J. V., Pette, D., Acetylcholinesterase mRNA level and synaptic activity in rat muscles depend on nerve-induced pattern of muscle activation. J. Neurosci. 1998, 18, 1944-1952.

[46] O'Reilly, C., Pette, D., Ohlendieck, K., Increased expression of the nicotinic acetylcholine receptor in stimulated muscle. Biochem. Biophys. Res. Comm. 2003, 300, 585-591.

[47] Glover, L., Quinn, S., Ryan, M., Pette, D., Ohlendieck, K., Supramolecular calsequestrin complex. Eur. J. Biochem. 2002, 269, 4607-4616.

[48] Pette, D., Staron, R. S., Mammalian skeletal muscle fiber type transitions. Int. Rev. Cytol. 1997, 170, 143-223.

[49] Bradford, M. M., A rapid and sensitive method for the quantitation of microgram quantities of protein utilizing the principle of protein-dye binding. Anal. Biochem. 1976, 72, 248254.

[50] Rabilloud, T., Valette, C., Lawrence, J. J., Sample application by in-gel rehydration improves the resolution of twodimensional electrophoresis with immobilized $\mathrm{pH}$ gradients in the first dimension. Electrophoresis 1994, 15, 1552-1558.

[51] Gorg, A., Postel, W., Gunther, S., Weser, J. et al., Approach to stationary two-dimensional pattern: influence of focusing time and immobiline/carrier ampholytes concentrations. Electrophoresis 1988, 9, 37-46.

[52] Dowling, P., Doran, P., Ohlendieck, K., Drastic reduction of sarcalumenin in Dp427 (dystrophin of $427 \mathrm{kDa}$ )-deficient fibres indicates that abnormal calcium handling plays a key role in muscular dystrophy. Biochem. J. 2004, 379, 479-488.

[53] Towbin, H., Staehelin, T., Gordon, J., Electrophoretic transfer of proteins from polyacrylamide gels to nitrocellulose sheets: Procedure and some applications. Proc. Natl. Acad. Sci. USA 1979, 76, 4350-4354.
[54] Gorg, A., Weiss, W., Dunn, M. J., Current two-dimensional electrophoresis technology for proteomics. Proteomics 2004, 4, 3665-3685.

[55] Domon, B., Aebersold, R., Mass spectrometry and protein analysis. Science 2006, 312, 212-217.

[56] Unlu, M., Morgan, M. E., Minden, J. S., Difference gel electrophoresis: A single gel method for detecting changes in protein extracts. Electrophoresis 1997, 18, 2071-2077.

[57] Tonge, R., Shaw, J., Middleton, B., Rowlinson, R., Rayner, S. et al., Validation and development of fluorescence twodimensional differential gel electrophoresis proteomics technology. Proteomics 2001, 1, 377-396.

[58] Marouga, R., David, S., Hawkins, E., The development of the DIGE system: 2D fluorescence difference gel analysis technology. Anal. Bioanal. Chem. 2005, 382, 669-678.

[59] Ohira, Y., Yoshinaga, T., Nomura, T., Kawano, F. et al., Gravitational unloading effects on muscle fiber size, phenotype and myonuclear number. Adv. Space Res. 2002, 30, 777-781.

[60] Carlson, B. M., Borisov, A. B., Dedkov, E. I., Khalyfa, A. et al., Effects of long-term denervation on skeletal muscle in old rats. J. Gerontol. 2002, 57, B366-B374.

[61] Vandervoort, A. A., Aging of the human neuromuscular system. Muscle Nerve 2002, 25, 17-25.

[62] Zhang, P., Chen, X., Fan, M., Signaling mechanisms involved in disuse muscle atrophy.. Med. Hypotheses 2007, 69, 310321.

[63] Heilig, A., Pette, D., Albumin in rabbit skeletal muscle. Origin, distribution and regulation by contractile activity. Eur. J. Biochem. 1988, 171, 503-508.

[64] Skorjanc, D., Jaschinski, F., Heine, G., Pette, D., Sequential increases in capillarization and mitochondrial enzymes in low-frequency-stimulated rabbit muscle. Am. J. Physiol. 1998, 274, C810-C818.

[65] Nishida, W., Kitami, Y., Abe, M., Hiwada, K., Gene cloning and nucleotide sequence of SM22 alpha from the chicken gizzard smooth muscle. Biochem. Int. 1991, 23, 663-668.

[66] Lepore, J. J., Cheng, L., Min Lu, M., Mericko, P. A. et al., Highefficiency somatic mutagenesis in smooth muscle cells and cardiac myocytes in SM22alpha-Cre transgenic mice. Genesis. 2005, 41, 179-184.

[67] Ono, S., Minami, N., Abe, H., Obinata, T., Characterization of a novel cofilin isoform that is predominantly expressed in mammalian skeletal muscle. J. Biol. Chem. 1994, 269, 15280-15286.

[68] Thirion, C., Stucka, R., Mendel, B., Gruhler, A. et al., Characterization of human muscle type cofilin (CFL2) in normal and regenerating muscle. Eur. J. Biochem. 2001, 268, 3473-3482.

[69] Nakashima, K., Sato, N., Nakagaki, T., Abe, H. et al., Two mouse cofilin isoforms, muscle-type (MCF) and non-muscle type (NMCF), interact with F-actin with different efficiencies. J. Biochem. (Tokyo) 2005, 138, 519-526.

[70] Neufer, P. D., Benjamin, I. J., Differential expression of Bcrystallin and Hsp27 in skeletal muscle during continuous contractile activity. Relationship to myogenic regulatory factors. J. Biol. Chem. 1996, 271, 24089-24095.

[71] Maier, A., Gorza, L., Schiaffino, S., Pette, D., A combined histochemical and immunohistochemical study on the dynamics of fast-to-slow fiber transformation in chronically stimulated rabbit muscle. Cell Tissue Res. 1988, 254, 59-68. 